\title{
Confinement of ECH Plasmas and Effect of Electrode Biasing in TOKASTAR-2*
}

\author{
Kazuhisa BABA, Kozo YAMAZAKI, Hideki ARIMOTO, Tetsutarou OISHI, Makoto HASEGAWA, \\ Motoki SUWABE and Tatsuo SHOJI \\ Department of Energy Engineering and Science, Graduate School of Engineering, \\ Nagoya University, Furo-cho, Chikusa-ku, Nagoya 464-8603, Japan
}

(Received 8 December 2010 / Accepted 4 May 2011)

\begin{abstract}
TOKASTAR-2 device is characterized by a hybrid configuration between tokamak and helical confinement systems with outboard helical field coils. The improvement of plasma confinement by $\boldsymbol{E} \times \boldsymbol{B}$ poloidal flow was tried by applying radial electric field with spherical electrode biasing, and the effect of outboard helical magnetic field application was investigated on these electrode biased plasmas. It is measured that plasma radial density profile was improved in spherical electrode biased case, but the density fluctuation including density collapse was induced. This type of fluctuation was reduced by the application of outboard helical magnetic field.
\end{abstract}

(C) 2011 The Japan Society of Plasma Science and Nuclear Fusion Research

Keywords: TOKASTAR-2, tokamak, helical system, hybrid configuration, spherical electrode biasing, radial electric field, $\boldsymbol{E} \times \boldsymbol{B}$ drift, outboard helical field, density fluctuation

DOI: $10.1585 /$ pfr.6.2402088

\section{Introduction}

Tokamak and helical systems have been widely recognized as efficient toroidal magnetic plasma confinement devices. Both of them have unique merits and demerits, and a tokamak-helical hybrid magnetic system called TOKASTAR (tokamak-stellarator) [1] was proposed to keep both of their merits and to compensate demerits for each other. In addition, it enables a low aspect ratio design which may result in a compact reactor design. We constructed a device named TOKASTAR-2 to evaluate the effect of external outboard helical field application on tokamak plasma confinement. The TOKASTAR-2 device used in this research has outboard helical field coils with the toroidal period $n=1$ or 2 outside eight toroidal field coils [2]. Main property of this device is to form tokamak and/or helical magnetic configuration by different type of coils. The coil shape was simplified by locating helical coils outboard as shown in Fig. 1. TOKASTAR-2 experiments are in the phase that plasma current induction has been attempted by the $\mathrm{OH}$ (Ohmic heating) coil system to optimize tokamak operation. As a pre-ionized phase of tokamak operation, plasmas without plasma current are initiated by the fundamental ECR (Electron Cyclotron Resonance) heating with microwave frequency of $2.45 \mathrm{GHz}$ [3].

Recently, we focus on the confinement property of the $\mathrm{ECH}$ plasma to explore the possibility of the formation of drift surfaces due to $\boldsymbol{E} \times \boldsymbol{B}$ poloidal flow.

This paper describes the study of the radial electric field formation by spherical electrode biasing [4]

author's e-mail: yamazaki@ees.nagoya-u.ac.jp

*) This article is based on the presentation at the 20th International Toki Conference (ITC20).

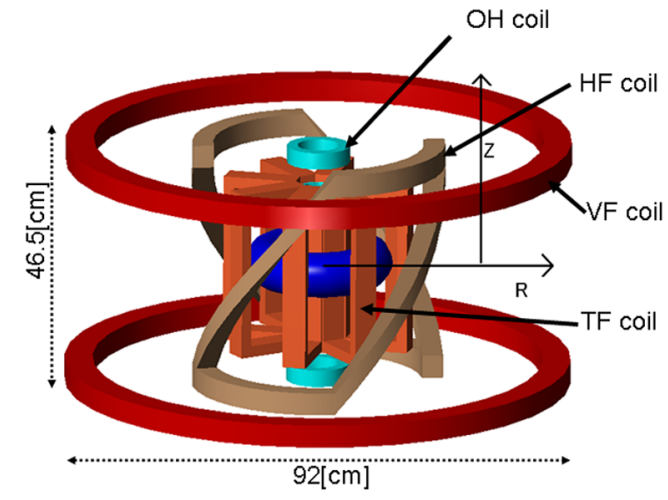

Fig. 1 Coil layout of TOKASTAR-2.

in order to improve plasma confinement property [5] in TOKASTAR-2 (Fig. 2). Firstly ECH plasma confinement in simple toroidal magnetic field was investigated before electrode biasing experiment. Next, radial profile of floating potential was measured to make sure radial electric field formation due to electrode biasing. At this point, floating potential dependence on bias voltage was examined, and then typical bias voltage was selected. Finally, radial electron density profile was measured, and density gradient length was evaluated. Improvement of plasma confinement by electrode biasing was evaluated by comparing them with no biasing case.

\section{ECH Plasma Initiation}

Plasmas were initiated by ECR heating without plasma current. In Fig. 3, top, middle and bottom signals show visible emission intensity of plasma measured 


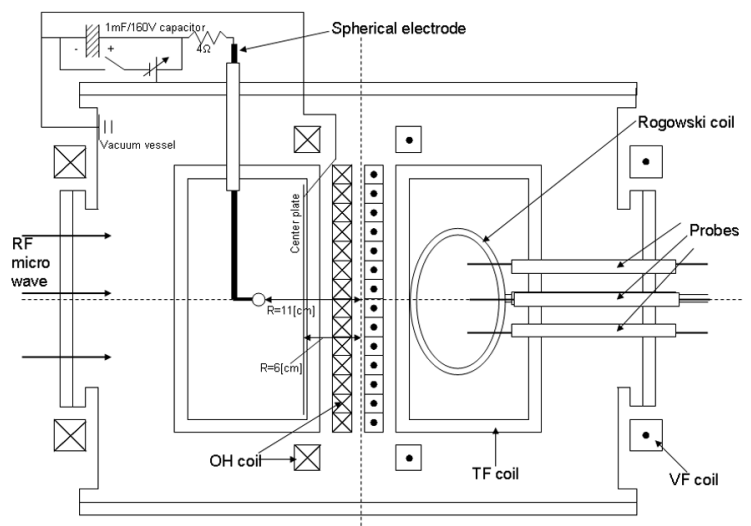

Fig. 2 Cross-section of device and instruments.

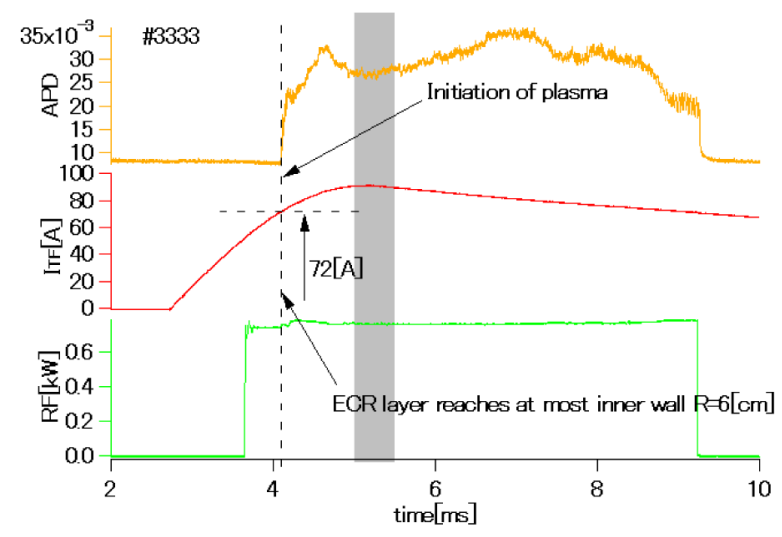

Fig. 3 Initiation of plasma by ECH.

by APD (Avalanche Photo Diode), toroidal coil conductor current, and RF injection power, respectively In this case, magnetic configuration was simple toroidal magnetic field formed by toroidal coils. Helium gas pressure was $0.5 \mathrm{~Pa}$ and injected RF power was $0.8 \mathrm{~kW}$. This figure shows that plasma was initiated when the toroidal coil current reached about $I_{\mathrm{TF}}=72 \mathrm{~A}$. The fundamental ECR layer reached near the inner wall and the plasma initiation by ECR was caused in this moment. Figure 4 shows contour map of toroidal field strength. Figure (a) is $x-y$ plan view on the mid plane $(Z[\mathrm{~cm}]=0)$, and figure (b) shows poloidal side view at toroidal angle $\varphi[\mathrm{deg}]=0$ (on the toroidal coil). In these figures, ECR layer $\left(B_{\mathrm{T}}=0.0875 \mathrm{~T}\right)$ was shown by red line. This ECR layer shifts outward with increasing toroidal coil current $I_{\mathrm{TF}}$. Plasma density profile on poloidal cross-section was shown in Fig. 5 when the toroidal field strength reached maximum in this case. The measured area of Fig. 5 is shown by gray color in Fig. 4(b). It is known that plasma heating was done outside of ECR layer in this time different from the plasma initiation period. Plasma electron density and temperature measured by Langmuir probes were approximately $n_{\mathrm{e}} \sim 10^{16} \mathrm{~m}^{-3}$ and $T_{\mathrm{e}} \sim 10 \mathrm{eV}$, respectively. Plasma current initiation trial by $\mathrm{OH}$ induction coil system also has been carried out for preparation of tokamak operation. However the present maximum plasma

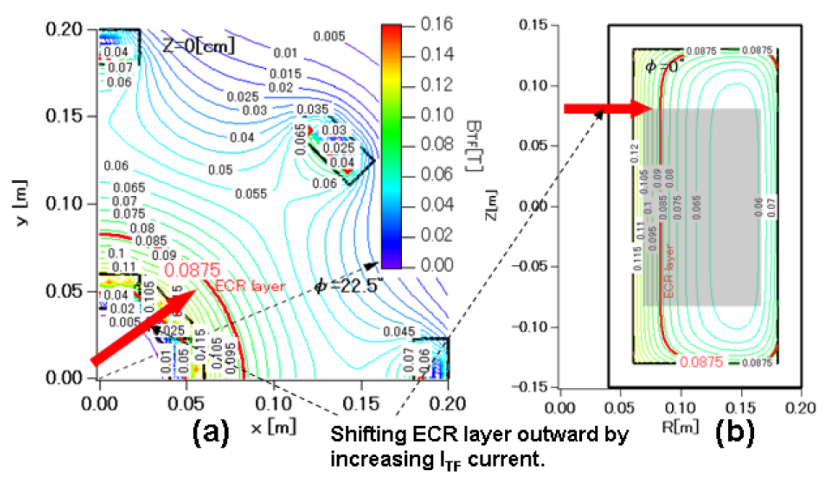

Fig. 4 Contour map of toroidal magnetic field strength. (a) $x$ $y$ horizontal cross-section. $(z=0 \mathrm{~cm})$, (b) $R-z$ vertical cross-section. $\left(\varphi=0^{\circ}\right)$.

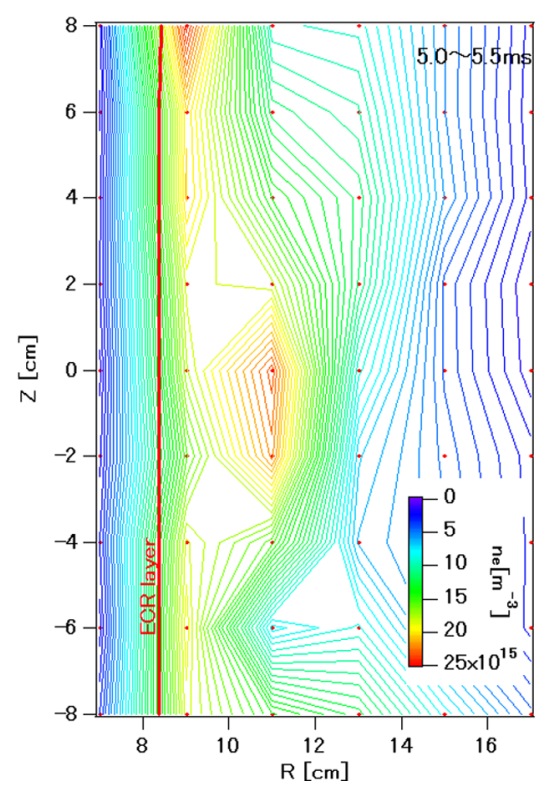

Fig. 5 Plasma density profile on poloidal cross-section. (RF inject port side)

current achieved was $I_{\mathrm{p}}=100 \mathrm{~A}$ during about $0.1 \mathrm{~ms}$. Order of $1 \mathrm{kA}$ plasma current is expected for stable low- $q$ tokamak operation.

\section{Spherical Electrode Biasing Exper- iment}

Radial electric field formation by spherical electrode biasing was attempted in order to improve plasma confinement property by $\boldsymbol{E} \times \boldsymbol{B}$ drift poloidal rotating flow in a simple toroidal magnetic field configuration. The spherical biasing electrode was set at $R=11 \mathrm{~cm}$ on mid plane $(Z[\mathrm{~cm}]=0)$ as shown in Fig. 2. The spherical electrode was constantly biased at $V_{\mathrm{e}}=+140 \mathrm{~V}$ against vacuum vessel during plasma discharge. The radial profile of plasma floating potential was measured by electro-static probes to confirm radial electric field formation. The ion saturation current $I_{\text {is }}$ was also measured for checking radial 


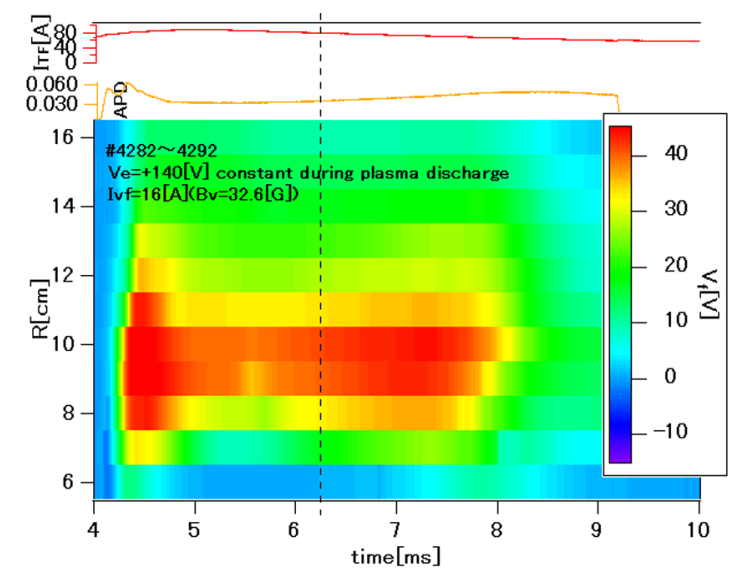

Fig. 6 Time evolution of radial plasma floating potential profile.

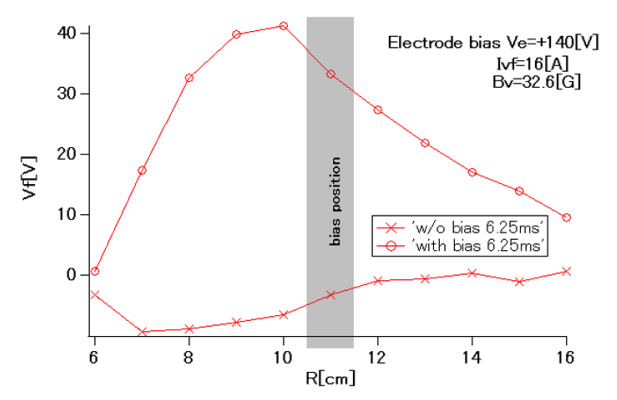

Fig. 7 Radial profile of plasma floating potential. Red crosses: without bias. Red circles: with bias.

electron density profile to evaluate the variation of plasma confinement property.

Figure 6 shows the time evolution of radial plasma floating potential profile in the biasing case. This figure shows that potential profile with peak value of about $V_{\mathrm{f}} \sim 40 \mathrm{~V}$ was formed at $R=9 \sim 10 \mathrm{~cm}$. This peaked potential profile continued for a long period during plasma existence. This clear peaked profile was obtained in case of vertical magnetic field application $B_{\mathrm{v}}=32.6 \mathrm{G}$ and not observed without vertical field. Radial floating potential profiles with biasing and without biasing at $t=6.25 \mathrm{~ms}$ (time shown by a broken line in Fig. 6) are shown in Fig. 7. Red crosses indicate the case without biasing, and red circles indicate the case with biasing. Potential well under $-10 \mathrm{~V}$ was measured in case without biasing. However, peaked potential of maximum about $+40 \mathrm{~V}$ was measured in case with biasing. Figure 8 shows the time evolution of radial electric field profile derived from potential gradient with biasing case. Radial electric field is formed inside and outside of $R=8 \sim 10 \mathrm{~cm}$. Figure 9 shows radial electric field profile with biasing case at $t=6.25 \mathrm{~ms}$. Radial electric field with biasing case is $E_{r} \sim 500 \mathrm{~V} / \mathrm{m}$ at outside of potential peak and $E_{r} \sim 1500 \mathrm{~V} / \mathrm{m}$ at inside, which are speculated from radial floating potential profile measurement. Figure 10 shows peak potential dependence on bias voltage. When the electrode is biased at positive voltage,

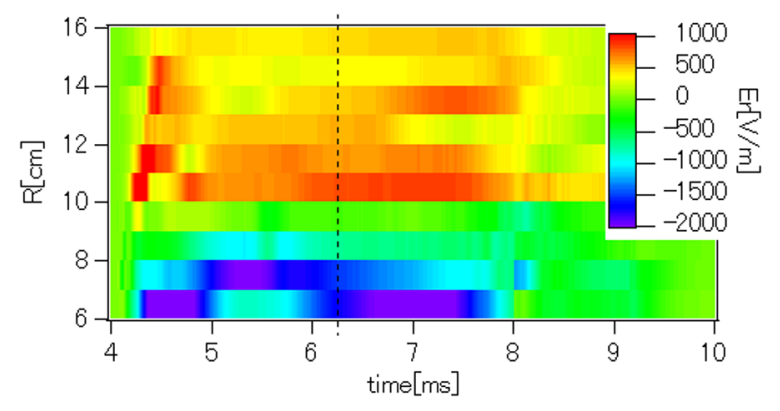

Fig. 8 Time evolution of radial electric field profile.

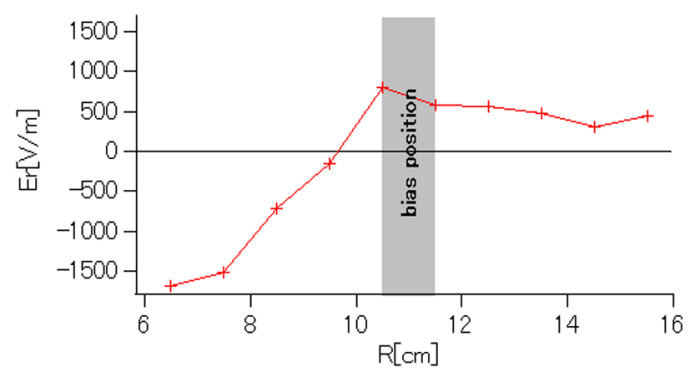

Fig. 9 Radial electric field profile at $t=6.25 \mathrm{~ms}$ shown in Fig. 8 .

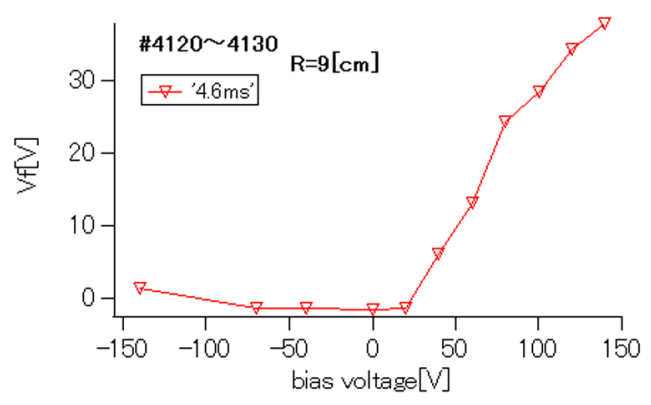

Fig. 10 Peak potential dependence on bias voltage. $(R=9 \mathrm{~cm})$

peak potential value $(R[\mathrm{~cm}]=9)$ increases proportional to the bias voltage. But, when the electrode is negatively biased, the measured potential is almost constant and is as same as no biasing case. It should be noted that electron plasma is initiated by ECH here. It could be considered that outward radial electron flux is formed in the negatively biased case so that plasmas profile with large potential gradient cannot be formed. When the electrode is biased at negative, it is valid technique that thermal electron is driven from hot cathode electrode. But, there is not thermal electron drive mechanics in biasing cold cathode electrode used in this experiment. Maximum voltage of the present electrode power supply is $+140 \mathrm{~V}$. Therefore, bias voltage $V_{\mathrm{e}}=+140 \mathrm{~V}$ was selected for detailed profile measurement.

Radial electron density profiles with biasing and without biasing at $t=6.25 \mathrm{~ms}$ are shown in Fig. 11. Red crosses indicate the case without biasing, and red circles indicate the case with biasing. The increases in electron density and density gradient can be confirmed with biasing case, 


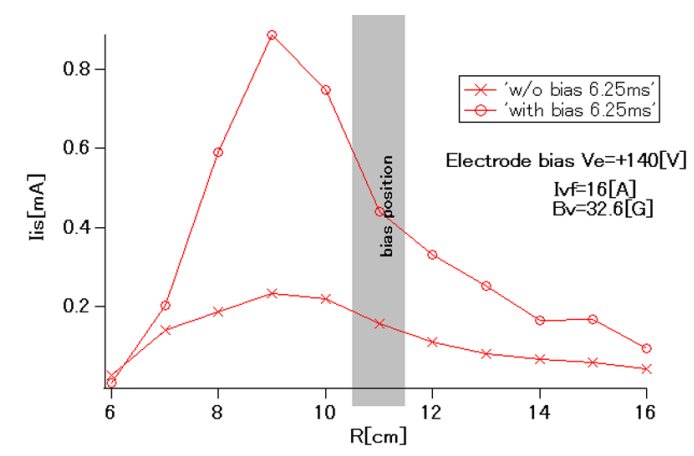

Fig. 11 Radial profile of electron density. Red crosses: without bias. Red circles: with bias.

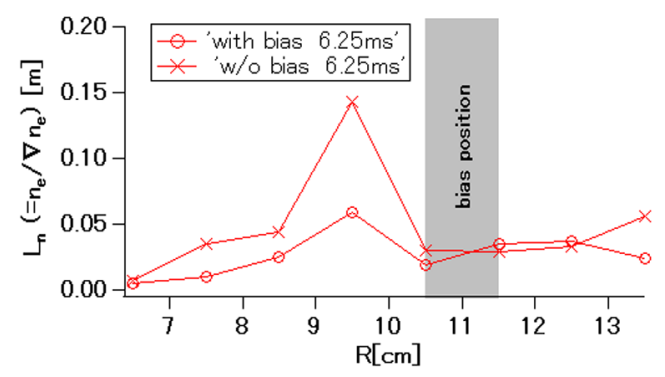

Fig. 12 Length of electron density gradient. Red crosses: without bias. Red circles: with bias.

which corresponds to plasma potential build-up. Figure 12 shows radial profile of electron density gradient length. When the electrode is biased, the length of density gradient becomes shorter than that without biasing case on the area of $R=7 \sim 11 \mathrm{~cm}$. Therefore, the confinement property is improved by electrode biasing at the inner area of bias position. It is expected that $\boldsymbol{E} \times \boldsymbol{B}$ poloidal flow forms drift surfaces contributing to the plasma confinement.

\section{Summary and Discussions}

ECH plasmas confined by simple toroidal magnetic field were produced. The formation of radial electric field by spherical electrode biasing was attempted for the improvement of plasma confinement property in simple toroidal magnetic field. In this experiment, plasma potential profile with peak of $+40 \mathrm{~V}$ was formed by biasing spherical electrode positive against vacuum vessel and applying upward or downward vertical field about $32.6 \mathrm{G}$. It could be expected that radial electric field was formed.

The electron density was peaked at $R=9 \mathrm{~cm}$ without biasing, and the increase in electron density centered at $R=9 \mathrm{~cm}$ were found in biased plasma case. The corresponding radial potential profiles were also measured. It is expected that plasma confinement was improved by the formation of drift surfaces due to $\boldsymbol{E} \times \boldsymbol{B}$ poloidal flow by radial electric field. The detailed mechanism will be clarified in the future.

There remain several future problems on the induction of plasma current without pulsed vertical field power supply. It is also necessary that contribution of electrode biasing to the plasma current induction would be examined.

[1] K. Yamazaki and Y. Abe, Research Report of the Institute of Plasma Physics, Nagoya, Japan, IPPJ-718 (1985).

[2] K. Yamazaki, Y. Taira, T. Oishi, H. Arimoto and T. Shoji, J. Plasma Fusion Res. SERIES 8, 1044 (2009).

[3] T. Oishi, K. Yamazaki, K. Okano, H. Arimoto, K. Baba, M. Hasegawa and T. Shoji, J. Plasma Fusion Res. SERIES 9, 69 (2010).

[4] S. Kitajima, J. Plasma Fusion Res. 82, 348 (2006).

[5] J. Fujita et al., IEEE Transaction on Plasma Science 9, 180 (1981). 